PROCEEDINGS OF THE

AMERICAN MATHEMATICAL SOCIETY

Volume 129, Number 3, Pages 921-930

S 0002-9939(00)05668-9

Article electronically published on September 20, 2000

\title{
THE COHOMOLOGY RINGS OF THE ORBIT SPACES OF FREE TRANSFORMATION GROUPS OF THE PRODUCT OF TWO SPHERES
}

\author{
RONALD M. DOTZEL, TEJ B. SINGH, AND SATYA P. TRIPATHI
}

(Communicated by Ralph Cohen)

\begin{abstract}
Let $G=Z_{p}, p$ a prime (resp. $S^{1}$ ), act freely on a finitistic space $X$ with $\bmod p$ (resp. rational) cohomology ring isomorphic to that of $S^{m} \times S^{n}$. In this paper we determine the possible cohomology algebra of the orbit space $X / G$.
\end{abstract}

\section{INTRODUCTION}

Let $G=Z_{p}, p$ a prime (resp. $S^{1}$, the circle group), act on a space $X$ with $\bmod p$ (resp. rational) cohomology ring isomorphic to that of $S^{m} \times S^{n}$; we abbreviate this as $X \sim_{p} S^{m} \times S^{n}$ (resp. $\left.X \sim_{Q} S^{m} \times S^{n}\right)$. There are two spaces associated with the transformation group $(G, X)$; viz. the fixed point set $X^{G}$ and the orbit space $X / G$. The homological nature of $X^{G}$ has been studied in detail by Adem 1], Bredon [3], Hsiang [4, Su [6] and Tomter [7. However, to our knowledge, no one has investigated the homological structure of the space $X / G$. We find here the possibilities for the cohomology algebra $H^{*}(X / G)$ when the action is free. Throughout this paper, we use Čech cohomology with coefficients in the field $F_{p}$ of $p$ elements or $Q$ of rational numbers, unless otherwise indicated. The $\bmod p$ Bockstein cohomology operation associated with the coefficient sequence $0 \rightarrow Z_{p} \rightarrow Z_{p^{2}} \rightarrow$ $Z_{p} \rightarrow 0$ will be denoted by $\beta$. We prove the following:

Theorem 1. Let $G=Z_{p}, p$ an odd prime, act freely on a finitistic space $X \sim_{p}$ $S^{m} \times S^{n}, 0<m \leq n$, and assume that $H^{*}(X ; Z)$ is of finite type. Then $H^{*}\left(X / G ; Z_{p}\right)$ is isomorphic to $Z_{p}[x, y, z] / \phi(x, y, z)$ as a graded commutative algebra, where $\phi(x, y, z)$ is one of the following graded ideals:

(i) $\left(x^{2}, y^{(m+1) / 2}, z^{2}\right), m$ odd, $\operatorname{deg} x=1, y=\beta(x), \operatorname{deg} z=n$;

(ii) $\left(x^{2}, y^{(m+n+1) / 2}, y^{(n-m+1) / 2} z-a y^{(n+1) / 2}, z^{2}-b y^{m}\right), m$ even, $n$ odd, $\operatorname{deg} x=$ $1, y=\beta(x), \operatorname{deg} z=m, a, b \in Z_{p}$ and $a=0$ necessarily when $n<2 m$;

(iii) $\left(x^{2}, y^{(n+1) / 2}, z^{2}-b y^{m}\right), n$ odd, $\operatorname{deg} x=1, y=\beta(x), \operatorname{deg} z=m, b \in Z_{p}, b \neq 0$ only when $m$ is even and $2 m<n$.

Theorem 2. Let $G=Z_{2}$ act freely on a finitistic space $X \sim_{2} S^{m} \times S^{n}, 0<m \leq n$. Then $H^{*}\left(X / G, Z_{2}\right)$ is isomorphic to $Z_{2}[y, z] / \psi(y, z)$ as a graded algebra, where $\psi(y, z)$ is one of the following graded ideals:

Received by the editors September 4, 1998 and, in revised form, June 3, 1999.

2000 Mathematics Subject Classification. Primary 57S17; Secondary 57S25. 
(i) $\left(y^{m+2}, z^{2}\right), \operatorname{deg} y=1, \operatorname{deg} z=n$;

(ii) $\left(y^{m+n+1}, y^{n-m+1}, z, z^{2}-a y^{m} z-b y^{2 m}\right), \operatorname{deg} y=1, \operatorname{deg} z=m, a, b \in Z_{2}$ and $a=0$ necessarily when $n<2 m$;

(iii) $\left(y^{n+1}, z^{2}-a y^{m} z-b y^{2 m}\right), \operatorname{deg} y=1, \operatorname{deg} z=m, a, b \in Z_{2}$ and $b=0$ necessarily when $m=n$ or $n<2 m$.

Theorem 3. Let $G=S^{1}$ act freely on a finitistic space $X \sim_{Q} S^{m} \times S^{n}, 0<m \leq$ $n$. Then $H^{*}(X / G ; Q)$ is isomorphic to $Q[y, z] / \psi(y, z)$ as a graded algebra, where $\psi(y, z)$ is one of the following graded ideals:

(i) $\left(y^{(m+1) / 2}, z^{2}\right), m$ odd, $\operatorname{deg} y=2, \operatorname{deg} z=n$.

(ii) $\left(y^{(m+n+1) / 2}, z y^{(n-m+1) / 2}-a y^{(n+1) / 2}, z^{2}-b y^{m}\right), m$ even, $n$ odd, $\operatorname{deg} y=$ $2, \operatorname{deg} z=m$ and $a=0$ necessarily when $n<2 m$.

(iii) $\left(y^{(n+1) / 2}, z^{2}-b y^{m}\right), n$ odd, $\operatorname{deg} y=2, \operatorname{deg} z=m, b \neq 0$ only when $m$ is even and $2 m<n$.

The main gadget employed in our proofs is the Leray-Serre spectral sequence of the fibration, $X \stackrel{\iota}{\rightarrow} X_{G} \stackrel{\pi}{\rightarrow} B_{G}$, which has $E_{2}^{k, l}=H^{k}\left(B_{G} ; H^{l}(X)\right)$ as its $E_{2^{-}}$ term and converges to $H^{k+l}\left(X_{G}\right)$, in the sense of Bredon [2], where the coefficients $H^{*}(X)$ are twisted by the action of $\pi_{1}\left(B_{G}\right)$ and $X_{G}=\left(E_{G} \times X\right) / G$ is the Borel construction of $X$ associated to a universal $G$-bundle $E_{G} \rightarrow B_{G}$. It can easily be seen that $X_{G}$ is paracompact, when $X$ is so.

\section{SOME KNOWN RESUlTS}

Suppose $G=Z_{p}, p$ a prime, acts on a finitistic space $X \sim_{p} S^{m} \times S^{n}$. The following facts can be easily deduced.

Proposition 1. If $G$ acts trivially on $H^{*}(X)$ and the spectral sequence of the map $\pi: X_{G} \rightarrow B_{G}$ degenerates, then $\sum_{k} \operatorname{rk} H^{k}\left(X^{G}\right)=4$ [2, VII, 1.6].

Proposition 2. If $m$ and $n$ are even and $p>2$, then $X^{G} \neq \Phi$ [2, III, 7.10].

Proposition 3. If $H^{*}(X ; Z)$ is of finite type, $p>2$ and $G$ acts nontrivially on $H^{*}(X)$, then $p=3$ and $X^{G} \neq \Phi[6]$.

Proposition 4. If $p=2$ and $G$ acts nontrivially on $H^{*}(X)$, then $X^{G} \neq \Phi$ and $m=n$ [2, VII, 7.5].

We recall that for $G=Z_{p}$,

$$
H^{*}\left(B_{G} ; Z_{p}\right)= \begin{cases}Z_{p}[t], & \operatorname{deg} t=1, p=2, \\ \bigwedge(s) \otimes Z_{p}[t], & \operatorname{deg} s=1, t=\beta(s), p>2,\end{cases}
$$

and for $G=S^{1}$,

$$
H^{*}\left(B_{G} ; Q\right)=Q[t], \quad \operatorname{deg} t=2
$$

\section{Proofs}

Proof of Theorem 1 . Since there are no fixed points, it follows from Propositions 1 , 2] and 3 that $m$ and $n$ cannot both be even and that $Z_{p}$ acts trivially on $H^{*}(X)$. Hence the Leray-Serre spectral sequence of the map $\pi: X_{G} \rightarrow B_{G}$ does not collapse at the $E_{2}$-term and $E_{2}^{k, l}=H^{k}\left(B_{G}\right) \otimes H^{l}(X)$. Let $r \geq 2$ be the smallest integer such that $d_{r} \neq 0$. By the multiplicative properties of the spectral sequence, we have $d_{r}\left(1 \otimes v_{1}\right) \neq 0$ or $d_{r}\left(1 \otimes v_{2}\right) \neq 0$. Suppose, first, that $d_{r}\left(1 \otimes v_{1}\right) \neq 0$. Then $r=m+1$ 
and $m$ must be odd. So we can write $d_{m+1}\left(1 \otimes v_{1}\right)=t^{(m+1) / 2} \otimes 1$. Now, we either have $d_{m+1}\left(1 \otimes v_{2}\right)=0$ or $n=m$ and $d_{m+1}\left(1 \otimes v_{2}\right)=a t^{m+1} \otimes 1,0 \neq a \in Z_{p}$. For $n \neq m$, obviously, $d_{m+1}\left(1 \otimes v_{2}\right)=0$ and $d_{m+1}\left(1 \otimes v_{3}\right)=t^{(m+1) / 2} \otimes v_{2}$. Thus the differentials

$$
\begin{aligned}
& d_{m+1}: E_{m+1}^{k, m} \rightarrow E_{m+1}^{k+m+1,0}, \text { and } \\
& d_{m+1}: E_{m+1}^{k, m+n} \rightarrow E_{m+1}^{k+m+1, n}
\end{aligned}
$$

are isomorphisms and we have $E_{\infty}=E_{m+2}$. Consequently, the only nonzero vector spaces in the $E_{\infty}$-term are $E_{\infty}^{k, n}=Z_{p}=E_{\infty}^{k, 0}, 0 \leq k \leq m$. Thus,

$$
H^{k}\left(X_{G}\right)= \begin{cases}Z_{p}, & 0 \leq k \leq m \text { and } n \leq k \leq m+n \\ 0, & \text { otherwise. }\end{cases}
$$

If $n=m$ and $d_{m+1}\left(1 \otimes v_{2}\right)=a t^{(m+1) / 2} \otimes 1, a \in Z_{p}$, then $d_{m+1}\left(1 \otimes v_{3}\right)=t^{(m+1) / 2} \otimes$ $\left(v_{2}-a v_{1}\right)$. So the differential

$$
d_{m+1}: E_{m+1}^{k, m} \rightarrow E_{m+1}^{k+m+1,0}
$$

is surjective with $\operatorname{ker}\left(d_{m+1}\right)$ generated by $\zeta_{k} \otimes\left(v_{2}-a v_{1}\right), \zeta_{k}$ is the generator of $H^{k}\left(B_{G}\right)$ and the differential

$$
d_{m+1}: E_{m+1}^{k, 2 m} \rightarrow E_{m+1}^{k+m+1, m}
$$

is injective with $i m\left(d_{m+1}\right)$ generated by $\zeta_{k+m+1} \otimes\left(v_{2}-a v_{1}\right)$. Consequently, $E_{\infty}=$ $E_{m+2}$ and the only nonzero entries in the $E_{\infty}$-term are $E_{\infty}^{k, m}=Z_{p}=E_{\infty}^{k, 0}$, for $0 \leq k \leq m$. We obtain

$$
H^{k}\left(X_{G}\right)= \begin{cases}Z_{p}, & 0 \leq k \leq 2 m \text { and } k \neq m \\ Z_{p} \oplus Z_{p}, & k=m \\ 0, & 2 m<k\end{cases}
$$

The multiplication by $t \in H^{2}\left(B_{G}\right)$ :

$$
t \cup(\cdot): E_{\infty}^{k, l} \rightarrow E_{\infty}^{k+2, l},
$$

regarded as a spectral sequence endomorphism, is an isomorphism for $0 \leq k \leq m-2$, $m>1$ and $l=0$ or $n$. If $m \neq n$ (resp. $m=n$ ) the element $1 \otimes v_{2}$ (respectively $\left.1 \otimes\left(v_{2}-a v_{1}\right)\right)$ of $E_{2}^{0, n}$ is a permanent cocycle and gives a nonzero element $w \in E^{0, n}$. There are elements $x \in H^{1}\left(X_{G}\right)$ and $y \in H^{2}\left(X_{G}\right)$ with $x=\pi^{*}(s)$ and $y=\pi^{*}(t)$. It is easily checked that the total complex

$$
\operatorname{Tot} E_{\infty}^{*, *} \cong Z_{p}[x, y, w] /\left(x^{2}, y^{(m+1) / 2}, w^{2}\right)
$$

as a graded commutative algebra. We choose an element $z \in H^{n}\left(X_{G}\right)$ such that $\iota^{*}(z)=v_{2}\left(\right.$ resp. $\left.v_{2}-a v_{1}\right)$ if $m \neq n($ resp. $m=n)$. Then $x z \neq 0 \neq y z$ and $z^{2}=0$. The multiplication

$$
y \cup(\cdot): H^{k}\left(X_{G}\right) \rightarrow H^{k+2}\left(X_{G}\right)
$$

is an isomorphism in degrees $k$ such that $0 \leq k \leq m-2$ and $n<k \leq m+n-2$. So

$$
H^{*}\left(X_{G}\right) \cong Z_{p}[x, y, z] /\left(x^{2}, y^{(m+1) / 2}, z^{2}\right) .
$$

For $m=1$, we have

$$
H^{*}\left(X_{G}\right) \cong Z_{p}[x, z] /\left(x^{2}, z^{2}\right)
$$


Since $G$ acts freely on $X, H^{*}\left(X_{G}\right)$ is isomorphic to $H^{*}(X / G)$ as a ring and we are in case (i).

Suppose, now, that $d_{r}\left(1 \otimes v_{1}\right)=0$ and $d_{r}\left(1 \otimes v_{2}\right) \neq 0$. We then have either $r=n-m+1$ and $d_{r}\left(1 \otimes v_{2}\right)=A \otimes v_{1}$ or $r=n+1$ and $d_{r}\left(1 \otimes v_{2}\right)=A \otimes 1$, $0 \neq A \in H^{*}\left(B_{G}\right)$. In the former case if $n$ were even, then we would have

$$
0=d_{r}\left(1 \otimes v_{2}^{2}\right)=A \otimes v_{1} v_{2}+(-1)^{n(1+r)} A \otimes v_{2} v_{1}=2 A \otimes v_{3} \neq 0 .
$$

Hence $n$ is odd. We then observe that $m$ must be even. Assume the contrary and consider the spectral sequence of the map $\pi$ with coefficients in $\mathbb{Z}$, the ring of integers. Since $H^{*}(X ; \mathbb{Z})$ is finitely generated, it has no $p$-torsion elements; consequently, we have $\widetilde{E}_{2}^{k, l}=H^{k}\left(B_{G} ; H^{l}(X ; \mathbb{Z})\right)=0$, for all $k$ odd. Thus $\widetilde{E}_{r}^{k, l}=0$, for all $k$ odd and $r \geq 2$. The coefficients homomorphism $q: \mathbb{Z} \rightarrow \mathbb{Z}_{p}$ gives the commutative diagram:

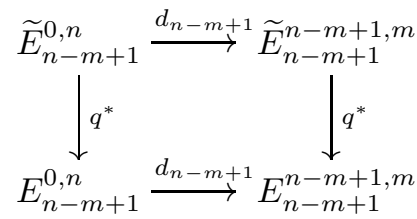

The composition $d_{n-m+1} \circ q^{*}$ is the trivial homomorphism, for $n-m+1$ is odd. Since $q^{*}$ in the left is surjective, the bottom $d_{n-m+1}$ is trivial. But this is not the case; hence our assertion. Thus we must have $m$ even and $n$ odd so that we can write $d_{n-m+1}\left(1 \otimes v_{2}\right)=t^{(n-m+1) / 2} \otimes v_{1}$. It follows that the differential

$$
d_{n-m+1}: E_{n-m+1}^{*, n} \rightarrow E_{n-m+1}^{*, m}
$$

is an isomorphism and $d_{n-m+1}\left(E_{n-m+1}^{*, m}\right)=0=d_{n-m+1}\left(E_{n-m+1}^{*, m+n}\right)$. So we have $E_{r}^{k, n}=0=E_{r}^{k+n-m+1, m}, E_{r}^{k, m+n}=E_{2}^{k, m+n}$ and $E_{r}^{k, 0}=E_{2}^{k, 0}$ for all $k \geq 0$ and $r=n-m+2$. It is easily seen that the differential

$$
d_{m+1}: E_{m+1}^{k, m} \rightarrow E_{m+1}^{k+m+1,0}
$$

is trivial for $0 \leq k \leq n-m$, since $E_{m+1}^{k, m}=E_{2}^{k, m}$. Because there are no fixed points, the differential

$$
d_{n+m+1}: E_{n+m+1}^{0, m+n} \rightarrow E_{n+m+1}^{n+m+1,0}
$$

must be nontrivial so that we can assume $d_{n+m+1}\left(1 \otimes v_{3}\right)=t^{(n+m+1) / 2} \otimes 1$. Then, the differential

$$
d_{n+m+1}: E_{n+m+1}^{*, m+n} \rightarrow E_{n+m+1}^{*, 0}
$$

is an isomorphism. Consequently, $E_{\infty}=E_{m+n+2}$ and the only nonzero vector spaces in the $E_{\infty}$-term are $E_{\infty}^{k, m}=Z_{p}$ for $0 \leq k \leq n-m$ and $E_{\infty}^{k, 0}=Z_{p}$ for $0 \leq k \leq m+n$. It follows that

$$
H^{k}\left(X_{G}\right)= \begin{cases}Z_{p}, & 0 \leq k<m \text { and } n<k \leq m+n \\ Z_{p} \oplus Z_{p}, & m \leq k \leq n \\ 0, & m+n<k .\end{cases}
$$

We note that $1 \otimes v_{1} \in E_{2}^{0, m}$ is a permanent cocycle and determines an element $w \in E_{\infty}^{0, m}$. We have

$$
\operatorname{Tot} E_{\infty}^{*, *} \cong Z_{p}[x, y, w] /\left(x^{2}, w^{2}, y^{(m+n+1) / 2}, y^{(n-m+1) / 2} w\right)
$$


as graded commutative algebras, where $x$ and $y$ satisfy $\pi^{*}(s)=x$ and $\pi^{*}(t)=y$. The multiplication

$$
y \cup(\cdot): H^{k}\left(X_{G}\right) \rightarrow H^{k+2}\left(X_{G}\right)
$$

is an isomorphism in degrees $k$ for $0 \leq k \leq n-2$ and $n<k \leq m+n-2$. We choose an element $z \in H^{m}(X / G)$ such that $\iota^{*}(z)=v_{1}$. Then $y^{r} z$ and $y^{(m+2 r) / 2}$ are linearly independent over $Z_{p}$ for $r \leq(n-m-1) / 2$. It is possible to change $z$ suitably so that $y^{(n-m+1) / 2} z=0$ and $z^{2}=b y^{m}, b \in Z_{p}$, when $n<2 m$ and $y^{(n-m+1) / 2} z=a y^{(n+1) / 2}$ and $z^{2}=b y^{m}, a, b \in Z_{p}$, when $2 m<n$. Therefore,

$$
H^{*}\left(X_{G}\right) \cong Z_{p}[x, y, z] /\left(x^{2}, y^{(m+n+1) / 2}, y^{(n-m+1) / 2} z-a y^{(n+1) / 2}, z^{2}-b y^{m}\right)
$$

and we are in case (ii).

Finally, consider the possibility $r=n+1, d_{r}\left(1 \otimes v_{1}\right)=0$ and $d_{r}\left(1 \otimes v_{2}\right)=A \otimes 1$, $0 \neq A \in H^{*}\left(B_{G}\right)$. Then $n$ must be odd and we can set $d_{n+1}\left(1 \otimes v_{2}\right)=t^{(n+1) / 2} \otimes 1$. So $d_{n+1}\left(1 \otimes v_{3}\right)= \pm t^{(n+1) / 2} \otimes v_{1}$; consequently the differentials

$$
\begin{aligned}
& d_{n+1}: E_{n+1}^{k, n} \rightarrow E_{n+1}^{k+n+1,0}, \\
& d_{n+1}: E_{n+1}^{k, m+n} \rightarrow E_{n+1}^{k+n+1, m}
\end{aligned}
$$

are isomorphisms. We obtain $E_{\infty}=E_{n+2}$ and the only nonzero vector spaces in the $E_{\infty}$-term are $E_{\infty}^{k, m}=Z_{p}=E_{\infty}^{k, 0}$ for $0 \leq k \leq n$. Thus

$$
H^{k}\left(X_{G}\right)= \begin{cases}Z_{p}, & 0 \leq k<m \text { and } n<k \leq m+n \\ Z_{p} \oplus Z_{p}, & m \leq k \leq n \\ 0, & m+n<k\end{cases}
$$

We note that $1 \otimes v_{1} \in E_{2}^{0, m}$ is, again, a permanent cocycle and gives an element $w \in E_{\infty}^{0, m}$. Choosing $x \in H^{1}\left(X_{G}\right)$ and $y \in H^{2}\left(X_{G}\right)$ such that $\pi^{*}(s)=x$ and $\pi^{*}(t)=y$, we obtain

$$
\operatorname{Tot} E_{\infty}^{*, *} \cong Z_{p}[x, y, w] /\left(x^{2}, y^{(n+1) / 2}, w^{2}\right)
$$

as graded commutative algebras. Now, we choose $z \in H^{m}\left(X_{G}\right)$ such that $\iota^{*}(z)=$ $v_{1}$. Then $z$ represents $w$ and the multiplication

$$
y \cup(\cdot): H^{k}\left(X_{G}\right) \rightarrow H^{k+2}\left(X_{G}\right)
$$

is an isomorphism for $0 \leq k \leq n-2$ and $n<k \leq m+n-2$, so that $y^{2} z \neq 0$ for $r \leq(n-1) / 2$. If $m$ is even and $n<2 m$, then $z^{2}=b y^{m / 2} z, b \in Z_{p}$. We replace $z$ by $z-(b / 2) y^{m / 2}$ and have $z^{2}=0$. If $m$ is even and $2 m<n$, then $z^{2}=b y^{m / 2} z+c y^{m}$, $b, c \in Z_{p}$. Replacing $z$ by $z-(b / 2) y^{m / 2}$ we obtain the relation $z^{2}=b^{\prime} y^{m}$. Thus

$$
H^{*}\left(X_{G}\right) \cong Z_{0}[x, y, z] /\left(x^{2}, y^{(n+1) / 2}, z^{2}-b y^{m}\right)
$$

as a graded commutative algebra and we obtain case (iii). This completes the proof of the theorem.

Proof of Theorem [2. The proof is analogous to that of Theorem 1] we describe it rather briefly. The spectral sequence of the map $\pi$ is nontrivial. Let $r \geq 2$ be the least integer such that $d_{r} \neq 0$. Then, we must have $d_{r}\left(1 \otimes v_{1}\right) \neq 0$ or 
$d_{r}\left(1 \otimes v_{2}\right) \neq 0$. Suppose that $r=m+1, d_{m+1}\left(1 \otimes v_{1}\right)=t^{m+1} \otimes 1$ and $m \neq n$. Then $d_{m+1}\left(1 \otimes v_{2}\right)=0, d_{m+1}\left(1 \otimes v_{3}\right)=t^{m+1} \otimes v_{2}$ so that $E_{\infty}=E_{m+2}$ and we obtain

$$
H^{k}\left(X_{G}\right)= \begin{cases}Z_{2}, & 0 \leq k \leq m \text { and } n \leq k \leq m+n \\ 0, & \text { otherwise. }\end{cases}
$$

The element $1 \otimes v_{2} \in E_{2}^{0, n}$, being a permanent cocycle, yields an element $w \in E_{\infty}^{0, n}$. So, the total complex Tot $E_{\infty}^{*, *}$ is the graded algebra $Z_{2}[y, w] /\left(y^{1}, w^{2}\right)$, where $y=$ $\pi^{*}(t), t \in H^{1}\left(B_{G}\right)$. Choose $z \in H^{n}\left(X_{G}\right)$ such that $\iota^{*}(z)=v_{2}$. Then $z$ determines $w$ and satisfies $z^{2}=0$. Since the multiplication

$$
y \cup(\cdot): H^{k}\left(X_{G}\right) \rightarrow H^{k+1}\left(X_{G}\right)
$$

is an isomorphism for $0 \leq k<m$ and $n \leq k<m+n$, we have $y^{r} z \neq 0,1 \leq r \leq m$. Therefore

$$
H^{*}\left(X_{G}\right) \cong Z_{2}[y, z] /\left(y^{m+1}, z^{2}\right) .
$$

As the action of $G$ is free, $H^{*}\left(X_{G}\right) \cong H^{*}(X / G)$ and we have the case (i). If $m=n, d_{m+1}\left(1 \otimes v_{1}\right)=t^{m+1} \otimes 1$ and $d_{m+1}\left(1 \otimes v_{2}\right)=c t^{m+1} \otimes 1, c \in Z_{2}$, then $d_{m+1}\left(1 \otimes v_{3}\right)=t^{m+1} \otimes\left(v_{2}+c v_{1}\right)$. We obtain $E_{\infty}=E_{m+2}$ and

$$
H^{k}\left(X_{G}\right)= \begin{cases}Z_{2}, & 0 \leq k \leq 2 m \text { and } k \neq m ; \\ Z_{2} \oplus Z_{2}, & k=m ; \\ 0, & 2 m<k .\end{cases}
$$

In this case, the element $1 \otimes\left(v_{2}+c v_{1}\right) \in E_{2}^{0, m}$ is a permanent cocycle and determines an element $w \in E_{\infty}^{0, m}$. Let $y \in H^{1}\left(X_{G}\right)$ and $z \in H^{m}\left(X_{G}\right)$ be such that $\pi^{*}(t)=y$ and $\iota^{*}(z)=v_{2}+c v_{1}$. Then $z$ represent $w$ in $E_{\infty}^{0, m}$ and satisfies $z^{2}=a y^{m} z$ for some $a \in Z_{2}$. The element $y z$ represents $0 \neq t w \in E_{\infty}^{1, m}$ and the multiplication

$$
y \cup(\cdot): H^{k}\left(X_{G}\right) \rightarrow H^{k+1}\left(X_{G}\right)
$$

is an isomorphism for $m<k<2 m$ so that $y^{r} z \neq 0,1 \leq r \leq m$. Thus

$$
H^{*}\left(X_{G}\right) \cong Z_{2}[y, z] /\left(y^{m+1}, z^{2}-a y^{m} z\right)
$$

and we are in case (iii) with $b=0$.

Next, when $d_{r}\left(1 \otimes v_{1}\right)=0, r=n-m+1$ and $d_{r}\left(1 \otimes v_{2}\right)=t^{r} \otimes v_{1}$, then $d_{r}(1 \otimes v)=Q$. So, we have $E_{r+1}^{k, m+n}=E_{2}^{k, m+n}, E_{r+1}^{k, 0}=E_{2}^{k, 0}$, for $k \geq 0$, and $E_{r+1}^{k, m}$ is trivial for $k>n-m$ and $E_{2}^{k, m}$ for $k \leq n-m$. It is easily seen that the differential

$$
d_{m+1}: E_{m+1}^{k, m} \rightarrow E_{m+1}^{k+m+1,0}
$$

is also trivial for $0 \leq k \leq n-m$. Then the differential

$$
d_{n+m+1}: E_{m+n+1}^{k, m} \rightarrow E_{m+n+1}^{k+m+n+1,0}
$$

must be an isomorphism for all $k$, because the action of $G$ on $X$ is free. Thus, the only nonzero vector spaces in the $E_{\infty}$-term are $E_{\infty}^{k, m}=Z_{2}$ for $0 \leq k \leq n-m$ and $E_{\infty}^{k, 0}=Z_{2}$, for $0 \leq k \leq m+n$. Consequently

$$
H^{k}\left(X_{G}\right)= \begin{cases}Z_{2}, & 0 \leq k<m \text { and } n<k \leq m+n \\ Z_{2} \oplus Z_{2}, & m \leq k \leq n \\ 0, & m+n<k\end{cases}
$$


The element $1 \otimes v_{1} \in E_{2}^{0, m}$ is a permanent cocycle and gives an element $w \in E_{\infty}^{0, m}$. We obtain

$$
\operatorname{Tot} E_{\infty}^{*, *} \cong Z_{2}[y, w] /\left(y^{m+n+1}, w^{2}, y^{n-m+1} w\right)
$$

as graded algebras. The multiplication by $\pi^{*}(t)=y$,

$$
y \cup(\cdot): H^{k}\left(X_{G}\right) \rightarrow H^{k+1}\left(X_{G}\right),
$$

is an isomorphism for $0 \leq k \leq n-1$ and $n<k<m+n$. Since the composition $\iota^{*} \pi^{*}$ is trivial in positive degrees, it is possible to choose $z \in H^{m}\left(X_{G}\right)$ such that $\iota^{*}(z)=v_{1}, y^{n-m+1} z=0$ and $z^{2}=a y^{m} z+b y^{2 m}$, where $a=0$ when $2 m>n$. Thus we have

$$
H^{*}\left(X_{G}\right) \cong Z_{2}[y, z] /\left(y^{m+n+1}, y^{n-m+1} z, z^{2}-a y^{m} z-b y^{2 m}\right)
$$

as graded algebras and we are in case (ii).

Finally, consider the case $d_{r}\left(1 \otimes v_{1}\right)=0, r=n+1$ and $d_{n+1}\left(1 \otimes v_{2}\right)=t^{n+1}$. Then $d_{n+1}\left(1 \otimes v_{3}\right)=t^{n+1} \otimes v_{1}$. So the differentials

$$
\begin{aligned}
& d_{n+1}: E_{n+1}^{k, n} \rightarrow E_{n+1}^{k+n+1,0}, \text { and } \\
& d_{n+1}: E_{n+1}^{k, m+n} \rightarrow E_{n+1}^{k+n+1, m}
\end{aligned}
$$

are isomorphisms. We have $E_{\infty}=E_{n+2}$ and the only nonzero vector spaces in the $E_{\infty}$-term are $E_{\infty}^{k, m}=Z_{2}=E_{\infty}^{k, 0}$, for $0 \leq k \leq n$. Therefore

$$
H^{k}\left(X_{G}\right)= \begin{cases}Z_{2}, & 0 \leq k<m \text { and } n<k \leq m+n \\ Z_{2} \oplus Z_{2}, & m \leq k \leq n \\ 0, & m+n<k\end{cases}
$$

Taking $y \in H^{1}\left(X_{G}\right)$ and $z \in H^{m}\left(X_{G}\right)$ with $\pi^{*}(t)=y$ and $\iota^{*}(z)=v_{1}$, it can be easily seen that

$$
H^{*}\left(X_{G}\right) \cong Z_{2}[y, z] /\left(y^{n+1}, z^{2}-a y^{m} z-b y^{2 m}\right),
$$

as graded algebras, where $b=0$, if $n<2 m$. This completes the proof.

Proof of Theorem 3 . Though arguments given herein are different, the technique of the proof remains the same. Since there are no fixed points, the spectral sequence of the map $\pi: X_{G} \rightarrow B_{G}$ does not collapse at the $E_{2}$-term and $E_{2}^{k, l}=H^{k}\left(B_{G}\right) \otimes$ $H^{l}(X)$, for the action on $H^{*}(X)$ is trivial. Let $r \geq 2$ be the integer such that $d_{r} \neq 0$. By the multiplicative properties of the spectral sequence, we have $d_{r}\left(1 \otimes v_{1}\right) \neq 0$ or $d_{r}\left(1 \otimes v_{2}\right) \neq 0$.

First, suppose that $d_{r}\left(1 \otimes v_{1}\right) \neq 0$. Then $r=m+1$ where $m$ is odd. We can write $d_{r}\left(1 \otimes v_{1}\right)=a t^{(m+1) / 2} \otimes 1,0 \neq a \in Q$. If $n=2 m, d_{m+1}\left(1 \otimes v_{2}\right)=b t^{(m+1) / 2} \otimes v_{1}$, then

$$
0=d_{m+1}\left(1 \otimes v_{2}^{2}\right)=2 b t^{(m+1) / 2} \otimes v_{1} v_{2} \neq 0,
$$

a contradiction. Therefore, either $d_{m+1}\left(1 \otimes v_{2}\right)=0$ or $m=n, d_{m+1}\left(1 \otimes v_{2}\right)=$ $b t^{m+1} \otimes 1, a \neq b \in Q$. In the first case, $d_{m+1}\left(1 \otimes v_{1} v_{2}\right)=a t^{(m+1) / 2} \otimes v_{2}$; so the differentials

$$
\begin{aligned}
& d_{m+1}: E_{m+1}^{k, m} \rightarrow E_{m+1}^{k+m+1,0}, \text { and } \\
& d_{m+1}: E_{m+1}^{k, m+n} \rightarrow E_{m+1}^{k+m+1, n}
\end{aligned}
$$


are isomorphisms and we have $E_{\infty}=E_{m+2}$. Thus, the only nonzero vector spaces in the $E_{\infty}$-term are $E_{\infty}^{k, n}=Q=E_{\infty}^{k, 0}, k$ even, $0 \leq k \leq m-1$. We have

$$
H^{k}\left(X_{G}\right)= \begin{cases}Q, & 0 \leq k \leq m-1, k \text { even; } n \leq k \leq n+m-1, k-n \text { even } \\ 0, & \text { otherwise. }\end{cases}
$$

In this case, $d_{m+1}\left(1 \otimes v_{2}\right)=b t^{(m+1) / 2} \otimes 1, b \neq 0, d_{m+1}\left(1 \otimes v_{1} v_{2}\right)=t^{(m+1) / 2} \otimes$ $\left(a v_{2}-b v_{1}\right)$. So the differential

$$
d_{m+1}: E_{m+1}^{k, m} \rightarrow E_{m+1}^{k+m+1,0}
$$

is surjective, with $\operatorname{ker}\left(d_{m+1}\right)$ generated by $\xi_{k} \otimes\left(a v_{2}-b v_{1}\right), \xi_{k}$ is the generator of $H^{k}\left(B_{G}\right)$ and the differential

$$
d_{m+1}: E_{m+1}^{k, 2 m} \rightarrow E_{m+1}^{k+m+1, m}
$$

is injective with $i m\left(d_{m+1}\right)$ generated by $\xi_{k+m+1} \otimes\left(a v_{2}-b v_{1}\right)$. Consequently, $E_{\infty}=$ $E_{m+2}$ and the nonzero $E_{\infty}$-terms are $E_{\infty}^{k, m}=Q=E_{\infty}^{k, 0}, 0 \leq k \leq m-1$ and $k$ even. We have

$$
H^{k}\left(X_{G}\right)= \begin{cases}Q, & 0 \leq k \leq m-1, k \text { even } ; m \leq k \leq 2 m-1, k \text { odd } \\ 0, & 2 m \leq k\end{cases}
$$

If $m=1$, then $H^{*}\left(X_{G}\right)=Q[z] /\left(z^{2}\right)$. So, we can assume that $m>1$. Then multiplication by $t \in H^{2}\left(B_{G}\right)$,

$$
t \cup(\cdot): E_{\infty}^{k, l} \rightarrow E_{\infty}^{k+2, l},
$$

regarded as a spectral sequence endomorphism, is an isomorphism, for $0 \leq k \leq m-2$ and $l=0, n$. If $m \neq n($ resp. $m=n)$ the element $1 \otimes v_{2}\left(\right.$ resp. $\left.1 \otimes\left(a v_{2}-b v_{1}\right)\right)$ of $E_{2}^{0, n}$ is a permanent cocycle and gives a nonzero element $w \in E_{\infty}^{0, n}$. Let $y=$ $\pi^{*}(t) \in H^{2}\left(X_{G}\right)$. Then the total complex

$$
\operatorname{Tot} E_{\infty}^{*, *} \cong Q[y, w] /\left(y^{(m+1) / 2}, w^{2}\right)
$$

as a graded commutative algebra. We choose an element $z \in H^{n}\left(X_{G}\right)$ such that $\iota^{*}(z)=v_{2}\left(\right.$ resp. $\left.a v_{2}-b v_{1}\right)$ if $m \neq n($ resp. $m=n)$. Then $y z \neq 0$ and $z^{2}=0$. The multiplication

$$
y \cup(\cdot): H^{k}\left(X_{G}\right) \rightarrow H^{k+2}\left(X_{G}\right)
$$

is an isomorphism in degrees $k$ such that $0 \leq k \leq m-2$ and $n \leq k \leq m+n-3$. So

$$
H^{*}\left(X_{G}\right) \cong Q[y, z] /\left(y^{(m+1) / 2}, z^{2}\right),
$$

as mentioned in case (i).

Next, suppose that $d_{r}\left(1 \otimes v_{1}\right)=0$ and $d_{r}\left(1 \otimes v_{2}\right) \neq 0$, where $r=n-m+1$. If $n$ is even, then $m$ must be odd. Consequently, $0=d_{n-m+1}\left(1 \otimes v_{2}^{2}\right)=2 a t^{q} \otimes v_{1} v_{2} \neq 0$, where $d_{r}\left(1 \otimes v_{2}\right)=a t^{q} \otimes v_{1}, 2 q=n-m+1$. Therefore $n$ is odd and $m$ is even. It follows that $d_{r}\left(1 \otimes v_{1} v_{2}\right)=0$ and the differential

$$
d_{r}: E_{r}^{*, n} \rightarrow E_{r}^{*, m}
$$

is an isomorphism. Thus

$$
E_{n-m+2}^{k, n}=0=E_{n-m+2}^{k+n-m+1, m}, \quad E_{n-m+2}^{*, m+n}=E_{2}^{*, m+n}, \quad E_{n-m+2}^{*, 0}=E_{2}^{*, 0} .
$$

Since $m$ is even,

$$
d_{m+1}: E_{m+1}^{k, m} \rightarrow E_{m+1}^{k+m+1,0}
$$


is trivial, for $0 \leq k \leq n-m$. Since there are no fixed points, the differential

$$
d_{n+m+1}: E_{n+m+1}^{0, m+n} \rightarrow E_{n+m+1}^{n+m+1,0}
$$

must be nontrivial so that we can assume $d_{n+m+1}\left(1 \otimes v_{1} v_{2}\right)=a t^{(n+m+1) / 2} \otimes 1$, $a \neq 0$. Then the differential

$$
d_{n+m+1}: E_{n+m+1}^{*, m+n} \rightarrow E_{n+m+1}^{*, 0}
$$

is an isomorphism. So $E_{\infty}=E_{n+m+2}$ and the nonzero vector spaces in the $E_{\infty^{-}}$ term are $E_{\infty}^{k, m}=Q, 0 \leq k \leq n-m$ and $E_{\infty}^{k, 0}=Q, 0 \leq k \leq m+n ; k$ even. We have

$$
H^{k}\left(X_{G}\right)= \begin{cases}Q, & 0 \leq k \leq m-1, n+1 \leq k \leq n+m-1, k \text { even } \\ Q \oplus Q, & m \leq k \leq n-1, k \text { even; } \\ 0, & \text { otherwise. }\end{cases}
$$

The element $1 \otimes v_{1} \in E_{2}^{0, m}$ is a permanent cocycle and determines an element $w \in E_{\infty}^{0, m}$. We see that

$$
\operatorname{Tot} E_{\infty}^{*, *} \cong Q[y, w] /\left(w^{2}, y^{(n+m+1) / 2}, w y^{(n-m+1) / 2}\right),
$$

as graded commutative algebras, where $y=\pi^{*}(t)$, as above. The multiplication

$$
y \cup(\cdot): H^{k}\left(X_{G}\right) \rightarrow H^{k+2}\left(X_{G}\right)
$$

is an isomorphism in degrees $k$, for $0 \leq k \leq n-2$ and $n+1 \leq k \leq n+m-3$. We find an element $z \in H^{m}\left(X_{G}\right)$ such that $\iota^{*}(z)=v_{1}$. Then $y^{r} z$ and $y^{(m+2 r) / 2}$ are linearly independent over $Q$, for $r \leq(n-m-1) / 2$. We can change $z$ suitably so that $z y^{(n-m+1) / 2}=0$ and $z^{2}=b y^{m}, b \in Q$, when $n<2 m$ and $z y^{(n-m+1) / 2}=a y^{(n+1) / 2}$ and $z^{2}=b y^{m}, a, b \in Q$, when $2 m<n$. Therefore,

$$
H^{*}\left(X_{G}\right) \cong Q[y, z] /\left(y^{(n+m+1) / 2}, z y^{(n-m+1) / 2}-a y^{(n+1) / 2}, z^{2}-b y^{m}\right)
$$

as mentioned in case (ii).

Finally, let us suppose that $d_{r}\left(1 \otimes v_{1}\right)=0, r=n+1$ and $d_{r}\left(1 \otimes v_{2}\right)=a t^{(n+1) / 2} \otimes 1$, $0 \neq a \in Q$. Then, $n$ must be odd. We have $d_{n+1}\left(1 \otimes v_{1} v_{2}\right)= \pm a t^{(n+1) / 2} \otimes v_{1}$; consequently the differentials

$$
\begin{aligned}
& d_{n+1}: E_{n+1}^{k, n} \rightarrow E_{n+1}^{k+n+1}, \text { and } \\
& d_{n+1}: E_{n+1}^{k, m+n} \rightarrow E_{n+1}^{k+n+1, m}
\end{aligned}
$$

are isomorphisms. We obtain $E_{\infty}=E_{n+2}$ and the only nonzero vector spaces in the $E_{\infty}$-term are $E_{\infty}^{k, m}=Q=e_{\infty}^{k, 0}, 0 \leq k \leq n, k$ even. Thus for $m$ even

$$
H^{k}\left(X_{G}\right)= \begin{cases}Q, & 0 \leq k \leq m-1, n+1 \leq k \leq n+m-1, k \text { even } \\ Q \oplus Q, & m \leq k \leq n, k \text { even; } \\ 0, & \text { otherwise }\end{cases}
$$

and for $m$ odd,

$$
H^{k}\left(X_{G}\right)= \begin{cases}Q, & 0 \leq k \leq m=1, k \text { even } ; m \leq k \leq m+n-1, k \text { odd } \\ 0, & \text { otherwise }\end{cases}
$$

The element $1 \otimes v_{1} \in E_{2}^{0, m}$ is a permanent cocycle and yields an element $w \in E_{\infty}^{0, m}$. We have

$$
\operatorname{Tot} E_{\infty}^{*, *} \cong Q[y, w] /\left(y^{(n+1) / 2}, w^{2}\right)
$$


as graded commutative algebras, where $y=\pi^{*}(t) \in H^{2}\left(X_{G}\right)$. Choose $z \in H^{m}\left(X_{G}\right)$ such that $\iota^{*}(z)=v_{1}$. Then $z$ represents $w$ and the multiplication

$$
y \cup(\cdot): H^{k}\left(X_{G}\right) \rightarrow H^{k+2}\left(X_{G}\right)
$$

is an isomorphism, for $0 \leq k \leq n-2$ and $n<k \leq m+n-2$. Accordingly, $y^{i} z \neq 0$ for $i \leq(n-1) / 2$. When $m$ is even, $z$ can be chosen so that $z^{2}=b y^{m / 2} z, b \in Q$, is zero for $n<2 m$. So

$$
H^{*}\left(X_{G}\right) \cong Q[y, z] /\left(y^{(n+1) / 2}, z^{2}-b y^{m}\right),
$$

as in case (iii).

Since $G$ acts freely on $X, H^{*}\left(X_{G}\right) \cong H^{*}(X / G)$ and this completes the proof.

Examples. An example of case (i) in Theorem 1 is obtained by considering the diagonal action of $G$ on $S^{m} \times S^{n}$ where $G$ acts freely on $S^{m}$ and trivially on $S^{n}$. In fact, this possibility can be put more succinctly as $X / G \sim_{p} L^{m} \times S^{n}$. A similar consideration gives examples of case (3), except when $2 m<n$ and $m$ is even. By the same method one obtains an example of case (i) in Theorem 2, which can be described as $X / G \sim_{2} \mathbb{R} P^{m} \times S^{n}$. The space $\mathbb{C} P^{(m-1) / 2} \times S^{n}$ has the cohomology of type (i) in Theorem [3 and is obtained by taking the diagonal action of $S^{1}$ on $S^{m} \times S^{n}$, where $S^{1}$ acts freely on $S^{m}$ and trivially on $S^{n}$. Similarly, case (iii) can also be illustrated.

\section{REFERENCES}

1. A. Adem, $Z / p Z$ actions on $\left(S^{n}\right)^{k}$, Trans. Amer. Math. Soc. 300 (1987), 791-809. MR 88b:57037

2. G. Bredon, Introduction to compact transformation groups, Academic Press, 1972. MR 54:1265

3. - Cohomological aspects of transformation groups, Proc. Conf. Trans. Groups, (New Orleans, 1967), Springer, 1968, pp. 245-280. MR 39:6303

4. W. Y. Hsiang, Cohomology theory of topological transformation groups, Springer, 1975. MR 54:1363 English transl. MR 83k:57029

5. J. McCleary, User's guide to spectral sequences, Publish or Perish, 1985. MR 87f:55014

6. J. C. Su, Periodic transformations on the product of two spheres, Trans. Amer. Math. Soc. 112 (1964), 369-380. MR 29:612

7. P. Tomter, Transformation groups on cohomology product of spheres, Invent. Math. 23 (1974), 79-88. MR 48:12510

Department of Mathematics, University of Missouri, St. Louis, Missouri 63121

E-mail address: dotzel@umsl.edu

Department of Mathematics, University of Delhi, Delhi-110007, India

E-mail address: crl@delnet.ren.nic.in

Department of Mathematics, University of Delhi, Delhi-110007, India 